IJMMS 32:1 (2002) 1-15

PII. S0161171202202239

http://ijmms.hindawi.com

(c) Hindawi Publishing Corp.

\title{
OPTIMAL CUSUM SCHEMES FOR MONITORING VARIABILITY
}

\author{
S. POETRODJOJO, M. A. ABDOLLAHIAN, and NARAYAN C. DEBNATH
}

Received 5 February 2002

\begin{abstract}
Cumulative Sum (Cusum) Control Schemes are widely used in industry for process and measurement control. Most Cusum applications have been in monitoring shifts in the mean level of a process rather than process variability. In this paper, we study the use of Markov chain approach in calculating the average run length (ARL) of a Cusum scheme when controlling variability. Control statistics $S$ and $S^{2}$, where $S$ is the standard deviation of a normal process, are used. The optimal Cusum schemes to detect small and large increases in the variability of a normal process are designed. The control statistic $S^{2}$ is then used to show that the Cusum scheme is superior to the exponentially weighted moving average (EWMA) in terms of its ability to quickly detect any large or small increases in the variability of a normal process. It is also shown that Cusum with control statistics sample variance $\left(S^{2}\right)$ and sample standard deviation $(S)$ perform uniformly better than those with control statistic $\log S^{2}$. Fast initial response (FIR) Cusum properties are also presented.
\end{abstract}

2000 Mathematics Subject Classification: 62P30.

1. Introduction. Since the 1950s, the problem of designing optimal control schemes has received considerable attention in the literature, see, for example, Rowlands et al. [21], Gan [9], and Crowder and Hamilton [6].

It is well known that Cusum procedures give tighter process control than the classical quality control schemes, such as Shewhart schemes. Another effective alternative to the Shewhart control chart is exponentially weighted moving average (EWMA) chart. The above two alternatives are especially effective for detecting relatively small shifts.

Many authors have contributed to the theory of Cusum and EWMA; see, for example, Page [16], Ewan and Kemp [8], Wortham and Ringer [23], Woodall [22], and Lucas and Saccucci [12]. However, the design of procedures to monitor or control the process variability appears to have attracted very little attention. Some exceptions are papers by Page [17], Bauer and Hackl [2, 3], Hawkins [10], Ng and Case [15], Ramirez [18], Domangue and Patch [7], Crowder and Hamilton [6], MacGregor and Harris [13], and Chang and Gan [5].

One of the purposes of this paper is to investigate the efficiency of the Markov chain approach in evaluating the ARL of a Cusum scheme designed to monitor the variability of a process.

The ARL profile of any given scheme is obtained by plotting the ARL against the percentage of increases in the variability. To confirm the efficiency of the Markov chain approach in calculating the ARL of the Cusum procedure, 10,000 simulation runs were carried out. The results of this comparisons together with the standard deviation for the simulation are presented in Tables 1.1 and 1.2. The results show 
TABLE 1.1. Comparisons of the ARL profile of Cusum using Rowlands' method (ROW), Markov chain approach (MC), and simulation (SIM) for sample size $n=2$. The control statistics $S$ and $S^{2}$ are used. The in control ARL is 200 .

\begin{tabular}{|c|c|c|c|c|c|c|}
\hline & $\sigma / \sigma_{T}$ & & 1.25 & 1.5 & 1.75 & 2.0 \\
\hline \multirow{10}{*}{$S$} & \multirow{3}{*}{ ROW } & opt. $h$ & 3.00 & 2.05 & 2.25 & 2.00 \\
\hline & & opt. $M$ & 1.03 & 1.25 & 1.19 & 1.26 \\
\hline & & $L_{1}$ & 31.13 & 13.37 & 8.16 & 5.68 \\
\hline & \multirow{4}{*}{ SIM } & opt. $h$ & 3.00 & 2.05 & 2.25 & 2.00 \\
\hline & & opt. $M$ & 1.03 & 1.25 & 1.19 & 1.26 \\
\hline & & $L_{1}$ & 31.18 & 13.38 & 8.02 & 5.66 \\
\hline & & $\sigma$ & 0.0740 & 0.0135 & 0.0042 & 0.0020 \\
\hline & \multirow{3}{*}{$\mathrm{MC}$} & opt. $h$ & 2.52 & 2.17 & 1.93 & 1.76 \\
\hline & & opt. $M$ & 1.11 & 1.21 & 1.29 & 1.36 \\
\hline & & $L_{1}$ & 33.05 & 13.52 & 7.98 & 5.62 \\
\hline \multirow{10}{*}{$S^{2}$} & \multirow{3}{*}{ ROW } & opt. $h$ & 11.20 & 9.17 & 8.18 & 7.52 \\
\hline & & opt. $M$ & 1.24 & 1.46 & 1.64 & 1.82 \\
\hline & & $L_{1}$ & 28.64 & 12.71 & 7.63 & 5.51 \\
\hline & \multirow{4}{*}{ SIM } & opt. $h$ & 11.20 & 9.17 & 8.18 & 7.52 \\
\hline & & opt. $M$ & 1.24 & 1.46 & 1.64 & 1.82 \\
\hline & & $L_{1}$ & 28.70 & 12.61 & 7.71 & 5.52 \\
\hline & & $\sigma$ & 0.0540 & 0.0099 & 0.0038 & 0.0019 \\
\hline & \multirow{3}{*}{ MC } & opt. $h$ & 11.21 & 9.14 & 8.09 & 7.41 \\
\hline & & opt. $M$ & 1.24 & 1.46 & 1.66 & 1.85 \\
\hline & & $L_{1}$ & 28.59 & 12.57 & 7.69 & 5.50 \\
\hline
\end{tabular}

that the ARL profile of Cusum using the Markov chain approach lies very close to that using Rowlands' method and those obtained by simulation, see Tables 1.1 and 1.2.

The basic idea of Rowlands' method is to utilize mean value theorem for integrals to establish expressions for the operating characteristic $p(0)$ and average sample number $N(0)$ of a single test and hence using the equation $\operatorname{ARL}(0)=N(0) /(1-p(0))$ to calculate average run length of a single sided decision interval scheme. A complete discussion of Rowlands' method can be found in [1].

We also give direction on how to design an optimal Cusum chart for monitoring process variability. The performance of the designed optimal Cusum chart is then compared with the EWMA suggested by Crowder and Hamilton [6] and with the Cusum and EWMA proposed by Chang and Gan [5], where statistic $\log \left(S^{2}\right)$ is used. It is also shown that the ARL profile of Cusum chart using control statistic $S^{2}$ is uniformly better than the ARL profile of the EWMA suggested by Crowder and Hamilton [6]. In addition, it is shown that the ARL profile of Cusum chart obtained using the Markov chain approach and control statistics $S$ and $S^{2}$ lies very closely to the ARL profile of the Cusum proposed by Chang and Gan [5], where they used control statistic $\log \left(S^{2}\right)$ to monitor process variability. 
TABLE 1.2. Comparisons of the ARL profile of Cusum using Rowlands' method (ROW), Markov chain approach (MC), and simulation (SIM) for sample size $n=3$. The control statistics $S$ and $S^{2}$ are used. The in control ARL is 200 .

\begin{tabular}{|c|c|c|c|c|c|c|}
\hline & $\sigma / \sigma_{T}$ & & 1.25 & 1.5 & 1.75 & 2.0 \\
\hline \multirow{10}{*}{$S$} & \multirow{3}{*}{ ROW } & opt. $h$ & 3.13 & 2.13 & 1.90 & 1.80 \\
\hline & & opt. $M$ & 1.50 & 1.71 & 1.78 & 1.82 \\
\hline & & $L_{1}$ & 20.14 & 8.18 & 5.04 & 3.55 \\
\hline & \multirow{4}{*}{ SIM } & opt. $h$ & 3.13 & 2.13 & 1.90 & 1.80 \\
\hline & & opt. $M$ & 1.50 & 1.71 & 1.78 & 1.82 \\
\hline & & $L_{1}$ & 20.08 & 8.22 & 4.84 & 3.47 \\
\hline & & $\sigma$ & 0.0262 & 0.0042 & 0.0013 & 0.0006 \\
\hline & \multirow{3}{*}{ MC } & opt. $h$ & 2.68 & 2.12 & 1.79 & 1.58 \\
\hline & & opt. $M$ & 1.57 & 1.71 & 1.82 & 1.92 \\
\hline & & $L_{1}$ & 20.49 & 8.14 & 4.81 & 3.41 \\
\hline \multirow{10}{*}{$S^{2}$} & \multirow{3}{*}{ ROW } & opt. $h$ & 13.59 & 10.50 & 9.13 & 9.13 \\
\hline & & opt. $M$ & 2.48 & 2.93 & 3.29 & 3.29 \\
\hline & & $L_{1}$ & 18.75 & 7.83 & 4.74 & 3.39 \\
\hline & \multirow{4}{*}{ SIM } & opt. $h$ & 13.59 & 10.50 & 9.13 & 9.13 \\
\hline & & opt. $M$ & 2.48 & 2.93 & 3.29 & 3.29 \\
\hline & & $L_{1}$ & 19.02 & 7.97 & 4.75 & 3.42 \\
\hline & & $\sigma$ & 0.0206 & 0.0036 & 0.0012 & 0.0006 \\
\hline & \multirow{3}{*}{ MC } & opt. $h$ & 13.67 & 10.60 & 9.08 & 8.12 \\
\hline & & opt. $M$ & 2.48 & 2.92 & 3.32 & 3.70 \\
\hline & & $L_{1}$ & 19.05 & 7.89 & 4.74 & 3.38 \\
\hline
\end{tabular}

2. Proposed Cusum control procedure. Consider a single-sided decision interval scheme where the lower and upper boundaries are placed at zero and $h(h>0)$, respectively. We assume that the quality of produced items is described by the value of a measurable characteristic $x$, where $x \sim N\left(\mu, \sigma^{2}\right)$ and $\sigma^{2}$ is a known constant. The observed values of random variable $x$ (i.e., $x_{i}, i=1,2,3, \ldots$ ) are assumed statistically independent.

We wish to control the value of $\sigma$ about its target value of $\sigma_{T}$. Samples of size $n$, $x_{j 1}, x_{j 2}, \ldots, x_{j n}$, are taken at regular time interval from the current production line and the sample variance for the $j$ th sample $S_{j}^{2}$ is defined by

$$
S_{j}^{2}=\sum_{i=1}^{n} \frac{\left(x_{j i}-\bar{x}_{j}\right)^{2}}{n-1} .
$$

For convenience, define the score by

$$
Y_{j}=\frac{n S_{j}^{2}}{\sigma_{T}^{2}} .
$$


For the in control situation $Y_{j} \sim \chi_{n-1}^{2}$, and for the out of control situation

$$
\frac{n S_{j}^{2}}{\sigma^{2}} \sim x_{n-1}^{2}
$$

that is,

$$
Y_{j}=\frac{n S_{j}^{2}}{\sigma^{2}} \frac{\sigma^{2}}{\sigma_{T}^{2}} \sim k^{2} \chi_{n-1}^{2}, \quad k=\frac{\sigma}{\sigma_{T}} .
$$

Thus for out of control situations $k>1$, while for in control situations $k=1$. We only consider the case where $k>1$, since a deterioration in the performance level of the process can only result in an increase in the variance.

In this paper, we use control statistics $S$ and $S^{2}$ as well as $\log \left(S^{2}\right)$ to monitor the variability of the process. Therefore, for simplicity we consider the score as

$$
V_{j}=\left(k^{2} Y_{j}\right)^{\delta},
$$

where $\delta=0.5$ and 1.0 will represent control statistics $S$ and $S^{2}$.

Define the Cusum sequence by

$$
Z_{N}=\max \left(0, Z_{N-1}+\left(V_{j}-M\right)\right)
$$

where $M$ is the reference value. We consider the case where $Z_{0}=0$ for Cusum without fast initial response (FIR) feature and the case where $Z_{0}>0$ for Cusum with FIR. If $Z_{N} \geq h$ for the first time, then the out of control signal will be given, where $N$ refers to the run length of the scheme.

3. Average run length calculation. Following the basic principle proposed by Brook and Evans [4], the interval between 0 and $h$ is divided into $t$-subintervals, where each subinterval has length $w=2 h /(2 t-1)$. This arrangement will create $t+1$ states of the Markov chain, namely, $E_{0}, E_{1}, \ldots, E_{t}$, where $E_{t}$ refers to the state in which the out of control signal is given, that is, the absorbing state of the chain. This transition matrix $\mathbf{R}$ is a $t \times t$ matrix and can easily be found using the method presented by Brook and Evans [4].

Let $p_{i j}$ denote the probability of moving from state $i$ to state $j$, then

$$
p_{i j}=\int_{a}^{b} f(\lambda) d \lambda
$$

where $\lambda_{i}=V_{j}-M, a=(j-i) w-0.5 w, b=(j-i) w+0.5 w$, and $f(\lambda)$ probability density function for the variable $\lambda$. Considering the fact that $n S^{2} / \sigma^{2} \sim \chi_{n-1}^{2}$ and applying the transformation rule, (3.1) can be written as

$$
p_{i j}=\int_{(a+M)(1 / \delta) / k}^{(b+M)(1 / \delta) / k} f(u) d u,
$$


where

$$
f(u)=\frac{1}{\Gamma((n-1) / 2) 2^{(n-1) / 2}} u^{(n-1) / 2-1} \exp \frac{(-u)}{2}
$$

(see the appendix).

The average run length and its other moments are calculated using the matrix $\mathbf{R}$ in the expressions given by Brook and Evans [4].

$$
\mathbf{v}^{m}=m\left\{(\mathbf{I}-\mathbf{R})^{-1} \mathbf{R}\right\} \mathbf{v}^{(m-1)} .
$$

Setting $m=1$ reduces (3.4) to

$$
\mathbf{v}=(\mathbf{I}-\mathbf{R})^{-1} \mathbf{1},
$$

where $\mathbf{I}$ is a $t \times t$ identity matrix, $\mathbf{1}$ is a column vector of ones, $\mathbf{R}$ is a $t \times t$ transition matrix, and $\mathbf{v}$ is a $t$ elements columns vector.

The ARL, given that the Cusum is initially in the $i$ th state, is the $i$ th element of ARL vector $\mathbf{v}$. The first element of vector $\mathbf{v}$ presents the ARL for the Cusum chart starting from zero. For FIR features where the Cusum chart neither start from zero nor always exactly start from the middle of a state we followed the idea of Lucas and Crosier [11] and used quadratic interpolation among the ARL's at three states closest to the state that contains $S_{0}$, that is, the starting point of the scheme.

4. Determination of parameters $M, h$, and $Z_{0}$. There are many schemes which have common in control ARL $\left(L_{0}\right)$, yet they have different values of $h$ and $M$; therefore, they have different out of control ARL $\left(L_{1}\right)$. This situation raises the question how to select the optimal $h$ and $M$, that is, the values of $h$ and $M$ which lead to the minimum out of control ARL for a given in control ARL. It is well known that for monitoring of the mean, the optimal reference value is $\Delta / 2$, where $\Delta$ is the shift desired to be detected, however for controlling the variability there is no common practice to choose the reference value of the scheme. Moustakides [14] has proved that the reference value of the sequential probability ratio test (SPRT) for a particular distribution is optimal.

Unfortunately, the distribution of the control statistics cannot always be specified; see, for example, Chang and Gan [5] who applied a particular algorithm to numerically find the optimal value of the reference value when considering $\log \left(S^{2}\right)$ as the control statistic, however, they applied SPRT method when $S^{2}$ is used as the control statistic.

Regula [19] proposed the following method for obtaining the optimal reference value for any Cusum schemes: let $f(y ; \theta)$ denote the probability density function of the scores, $\theta$ being the parameter to be controlled. If $\theta=\theta_{0}$ represents the in control value and $\theta=\theta_{1}$ is the value of which the process is to be judged out of control, then the optimal reference value is obtained by solving the equation

$$
\frac{f\left(y ; \theta_{0}\right)}{f\left(y ; \theta_{1}\right)}=1
$$

that is, the value of $y$ which makes the ratio of the two densities equal to unity.

Regula [19] only considered Gamma family distribution and could only prove his result for the special case of the exponential distribution when the decision interval $h$ was smaller than the reference value $M$. 
TABLE 4.3. Comparisons of the ARL profile of optimal Cusum chart obtained using Regula's procedure and optimal Cusum designed by Chang and Gan (C-G) when statistic $\log S^{2}, n=5$, and $Z_{0}=0$ are used.

\begin{tabular}{lllllll}
\hline & C-G* & Cusum & C-G* & Cusum & C-G* & Cusum \\
$\sigma / \sigma_{T}$ & $M=0.309$ & $M=0.2509$ & $M=0.391$ & $M=0.3177$ & $M=0.451$ & $M=0.3782$ \\
& $h=1.210$ & $h=1.3797$ & $h=1.014$ & $h=1.1867$ & $h=0.896$ & $h=1.0418$ \\
& $\sigma_{\mathrm{opt}}=1.30$ & $\sigma_{\mathrm{opt}}=1.30$ & $\sigma_{\mathrm{opt}}=1.40$ & $\sigma_{\mathrm{opt}}=1.40$ & $\sigma_{\mathrm{opt}}=1.50$ & $\sigma_{\mathrm{opt}}=1.50$ \\
\hline 1.00 & 100 & 100 & 100 & 100 & 100 & 100 \\
1.01 & 86.6 & 86.3420 & 86.9 & 86.6181 & 87.3 & 86.8932 \\
1.02 & 75.4 & 74.9781 & 76.1 & 75.4548 & 76.5 & 75.9179 \\
1.03 & 66.0 & 65.5196 & 66.8 & 66.1135 & 67.5 & 66.6903 \\
1.04 & 58.2 & 57.5988 & 59.0 & 58.2523 & 59.7 & 58.8905 \\
1.05 & 51.5 & 50.9267 & 52.4 & 51.6004 & 53.2 & 52.2637 \\
1.10 & 30.2 & 29.7283 & 30.9 & 30.2574 & 31.6 & 30.7987 \\
1.20 & 13.8 & 13.6729 & 14.1 & 13.8201 & 14.4 & 14.0251 \\
1.30 & 8.15 & 8.1760 & 8.20 & 8.1513 & 8.31 & 8.1881 \\
1.40 & 5.63 & 5.7164 & 5.59 & 5.6233 & 5.61 & 5.5887 \\
1.50 & 4.29 & 4.3994 & 4.21 & 4.2801 & 4.19 & 4.2155 \\
2.00 & 2.11 & 2.2104 & 2.01 & 2.0967 & 1.97 & 2.0248 \\
\hline
\end{tabular}

* The numbers are from Chang and Gan [5].

By intensive numerical work, Abdollahian [1] showed that for control of the variability as well as control of mean, the optimal reference value obtained by applying an optimization procedure similar to Chang and Gan [5] fully supports Regula's proposal without any condition on the value of $h$. Abdollahian [1] considered control statistics $S, S^{1.5}, S^{2}, S^{3}$, and $S^{4}$. In this paper, we used Regula's proposal to obtain the optimal reference value for the Cusum scheme compatible with the one proposed by Chang and Gan [5], where they used a lengthy optimization procedure to obtain the optimal reference value. The results indicate that the optimal Cusum designed using Regula's procedure has ARL profile very close to those obtained by Chang and Gan [5], yet it is simpler to design, see Table 4.3.

It is worth mentioning that using $S^{2}$ as the control statistic, Regula's method is identical to SPRT, since $S^{2}$ follows the chi-squared distribution, on the other hand, since $S$ has no specific distribution, Regula's method is a simple reliable method to obtain the optimal reference value.

In this paper, we also investigate the performance of the Cusum schemes with FIR feature. Following the original idea of Lucas and Crosier [11] where they used $Z_{0}=h / 2$ as the head start when controlling the mean, we use $Z_{0}=h / 2$ when monitoring the variability.

5. Accuracy and related results. To determine the ARL of a one-sided Cusum chart using a Markov chain approach, most authors apply the least squares approximation, that is,

$$
\operatorname{ARL}(t)=\text { Asymptotic ARL }+\frac{B}{t}+\frac{C}{t^{2}}
$$


or

$$
\operatorname{ARL}(t)=\text { Asymptotic ARL }+\frac{B}{t^{2}}+\frac{C}{t^{4}} .
$$

Reynolds et al. [20] used the Markov chain approach to monitor the mean and observed several inconsistencies when applying the least squares approximation, specially for moderate value of $t$. Lucas and Crosier [11] concluded that "although there would be some unexpected results when applying the least squares procedure, in all cases the difference between the asymptotic ARL and the ARL obtained by using integral equation approach is less than 3\% when controlling the mean." We observed similar phenomenon when the Markov chain approach was used to monitor the variability.

To overcome this problem we used Markov chain procedure with 100 transient states $(t=100)$ to calculate the ARL rather than applying the least squares procedure. The results for the control of variability indicate a precision similar to that given by Lucas and Crosier [11].

6. Numerical results and comparison. The performance of the schemes in this paper are assessed based on their ARL. For a given in control ARL, the scheme with the minimum out of control ARL is called the optimal scheme.

Chang and Gan [5] provided extensive tables of comparison among schemes. They proposed a Cusum scheme using control statistic $\log S^{2}$ and found that their proposal is superior to the Cusum scheme using control statistic $S^{2}$ when monitoring process variability. One of the advantages of Cusum using $\log S^{2}$ is that it is possible to construct control schemes for the high sided as well as the low sided (two sided scheme). Hence, we can also monitor the possibility of quality improvement in any process industry. They also showed that their scheme performs nearly better than the EWMA proposed by Crowder and Hamilton [6]. However, if $S^{2}$ is used, we can only construct the high sided scheme.

In order to calculate ARL for Cusum when monitoring process variability, we can use both $S^{2}$ and $\log S^{2}$. The computed ARLs are very similar for a given shift in variability. However, if it is desired to detect small shifts in process variability when sample size is small ( $n=2$ and $n=3$ ), Chang and Gun [5] discovered that, numerically is impossible to use $\log S^{2}$ especially when in control ARL is large. On the other hand, if we use control statistic $S^{2}$, then it is possible to calculate the ARL in such condition by increasing the number of transient states in the Markov chain, however, this will increase the computation time substantially, thus it is not practical.

If the sample size is moderately large (more than 5), the ARL can be calculated using Markov chain approximation for any required shift. To overcome the problem of calculating ARL when sample size is small, we have considered monitoring variability using Cusum scheme with control statistic $S$. The complete comparisons of Cusum of $S^{2}$ and Cusum of $S$ together with Cusum of $\log S^{2}$ proposed by Chang and Gan [5] are given in Tables 5.4, 5.5, and 5.6.

From these tables, clearly we can see that Cusum using control statistic $S$ performs as good as Cusum using control statistic $\log \left(S^{2}\right)$, while Cusum using control statistic $S^{2}$ performs significantly better than the other two schemes. 


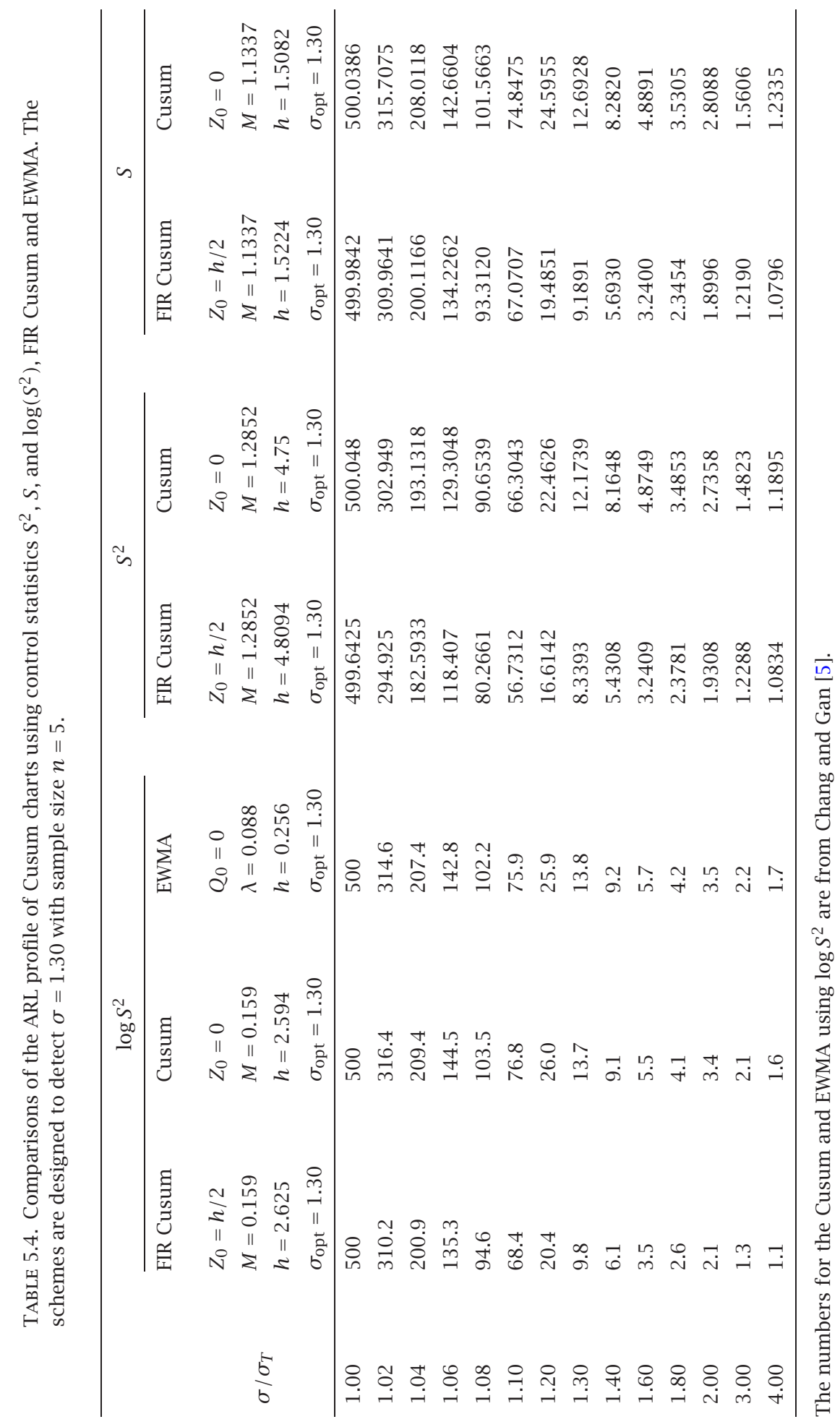


OPTIMAL CUSUM SCHEMES FOR MONITORING VARIABILITY

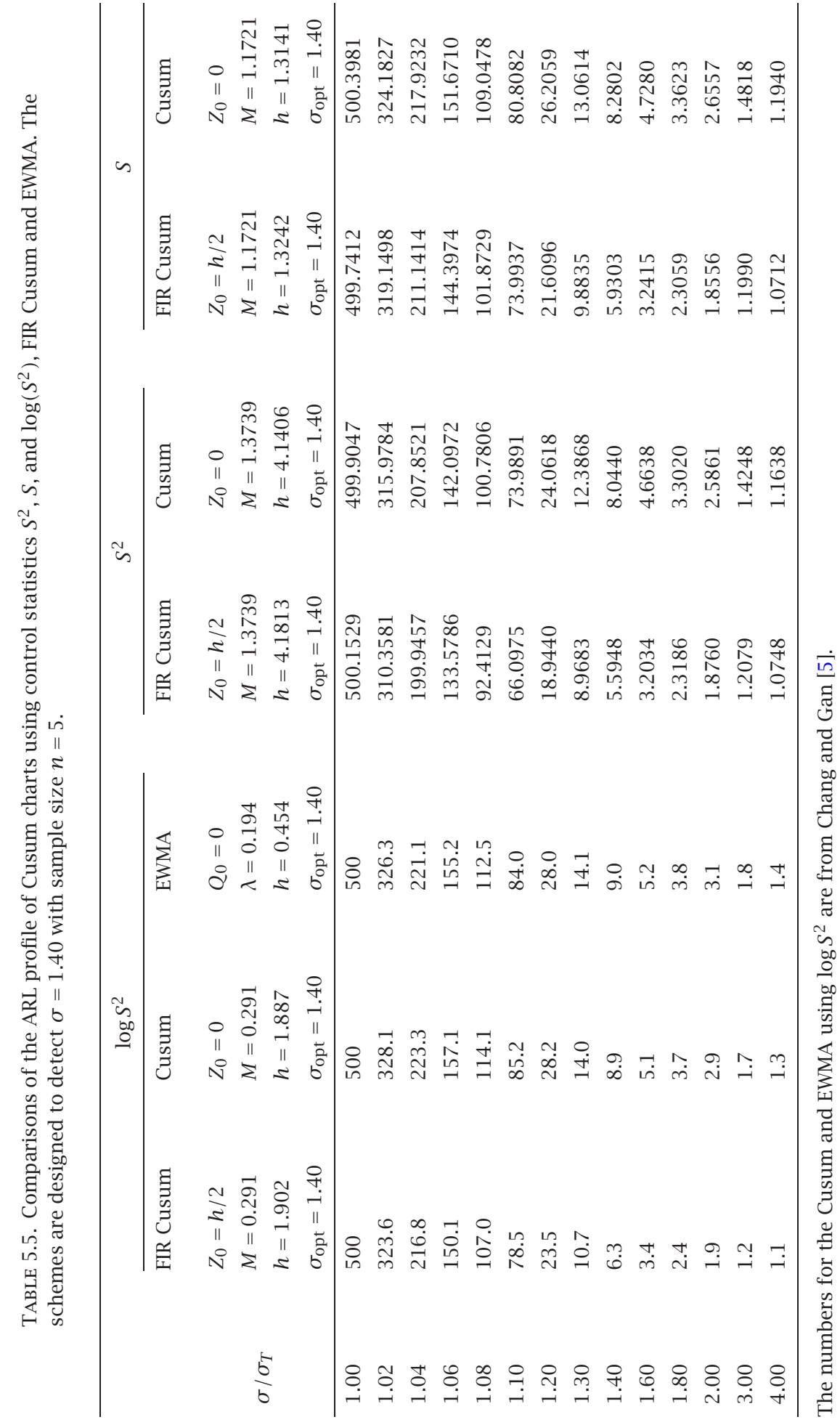


As was mentioned earlier, we would experience numerical problems in detecting small shifts by using the control statistic $S^{2}$, when only small samples are available. These problems do not exist when we consider control statistic $S$. Thus, Cusum with control statistic $S$ is the appropriate alternative choice when it is desired to monitor a small shifts using small sample ( $n=2$ or $n=3)$. To provide an assessment on the performance of Cusum using control statistic $S$ when monitoring small shifts in process variability with small samples ( $n=2$ and $n=3$ ), we have compared the ARL profiles of the Cusum with the simulations results based on 10,000 simulated runs. The results of the simulations together with the standard deviation for the simulations are presented in Tables 7.7 and 7.8. The results show that the ARL profile of the proposed Cusum lies very closely to the ARL profile produced by the simulation, confirming the effectiveness of the control statistic $S$ for monitoring small shifts in process variability when small sample is available.

7. Conclusions. In this paper, a one sided Cusum procedure for monitoring process variability is presented. The ARL is obtained using Markov chain approximation. It is shown that Markov chain procedure is comparable with other procedures; such as, Integral equation approach and Simulation when calculating the ARL for Cusum scheme designed to monitor variability of a process. Therefore, Markov chain approach is used throughout the paper to calculate ARL of the Cusum scheme. It is proposed to use Regula's method to obtain the optimal reference value for Cusum, when monitoring process variability. It is shown that ARL profile of optimal Cusum obtained using Regula's method lies very closely to those suggested by Chang and Gan [5] and yet is simpler to design.

The paper also investigates the effectiveness of control statistics $S, S^{2}$, and $\log \left(S^{2}\right)$ for monitoring process variability. It is shown that control statistic $S^{2}$ outperforms the other two control statistics.

Finally we have designed optimal Cusum and FIR Cusum by using Regula's method to obtain optimal reference value and Markov chain approximation to calculate their ARLs. The control statistics used are $S$ and $S^{2}$. The ARL profile for the two schemes using control statistics $S$ and $S^{2}$ are compared with those given by Chang and Gan [5] where they have used control statistic $\log \left(S^{2}\right)$ to monitor variability and optimization procedure to obtain optimal reference value. The results confirm the fact that our proposed optimal Cusum and FIR Cusum charts outperform the optimal Cusum and EWMA charts suggested by Chang and Gan [5]. The results also indicate that Cusum chart performs better than EWMA chart when monitoring process variability regardless of the control statistics used.

To overcome the problem of detecting small shifts in process variability with small sample sizes, it is proposed to use Cusum and FIR Cusum using control statistic $S$. Then the ARL for the latter schemes are obtained using Markov chain approach. The numerical results show that both Cusum and FIR Cusum produce ARL profiles that lie very closely to simulation results indicating that it is possible to design Cusum schemes aimed to monitor small process variability when only small sample size is available, if Markov chain approach is used to calculate the ARLs. 


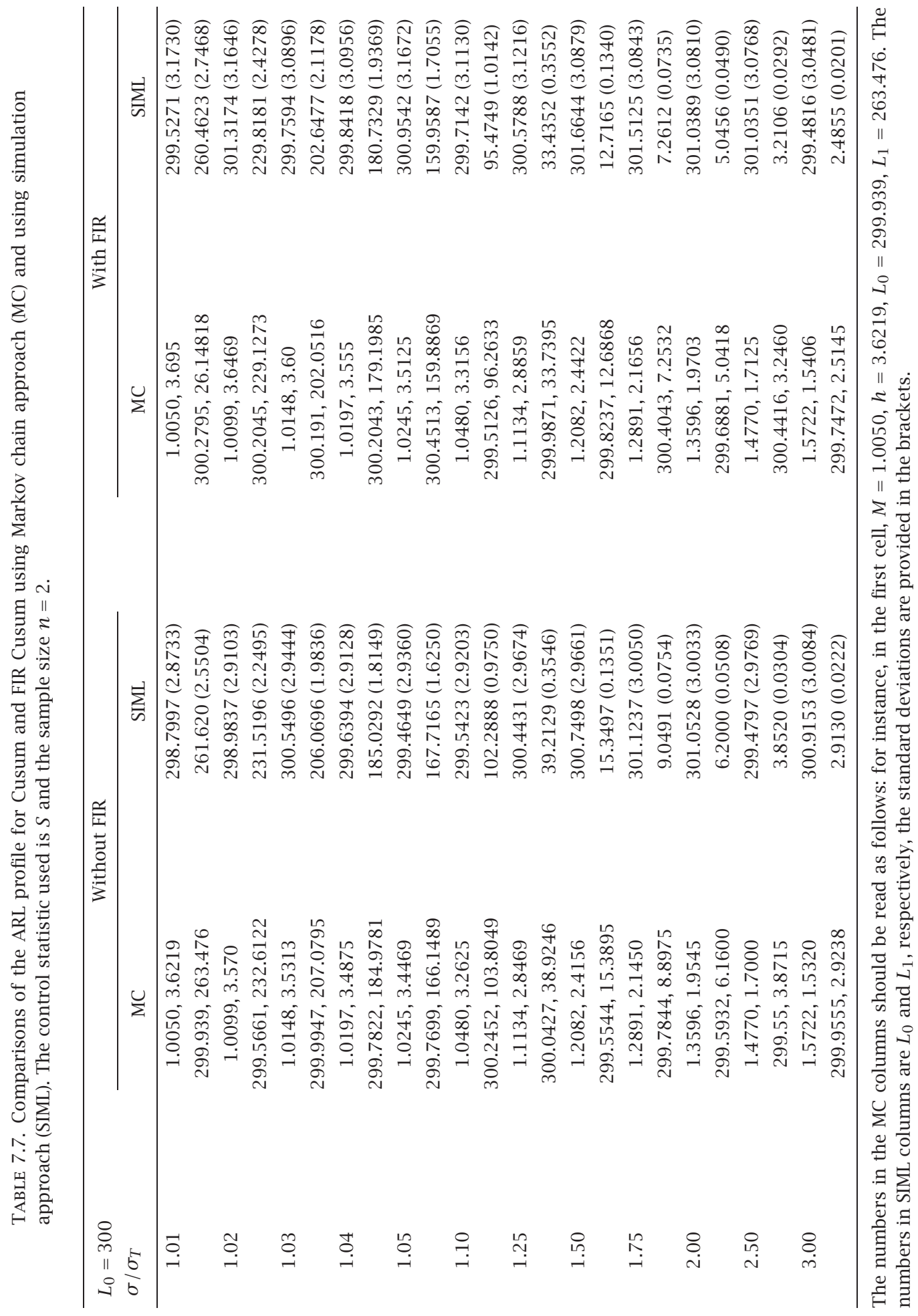


OPTIMAL CUSUM SCHEMES FOR MONITORING VARIABILITY

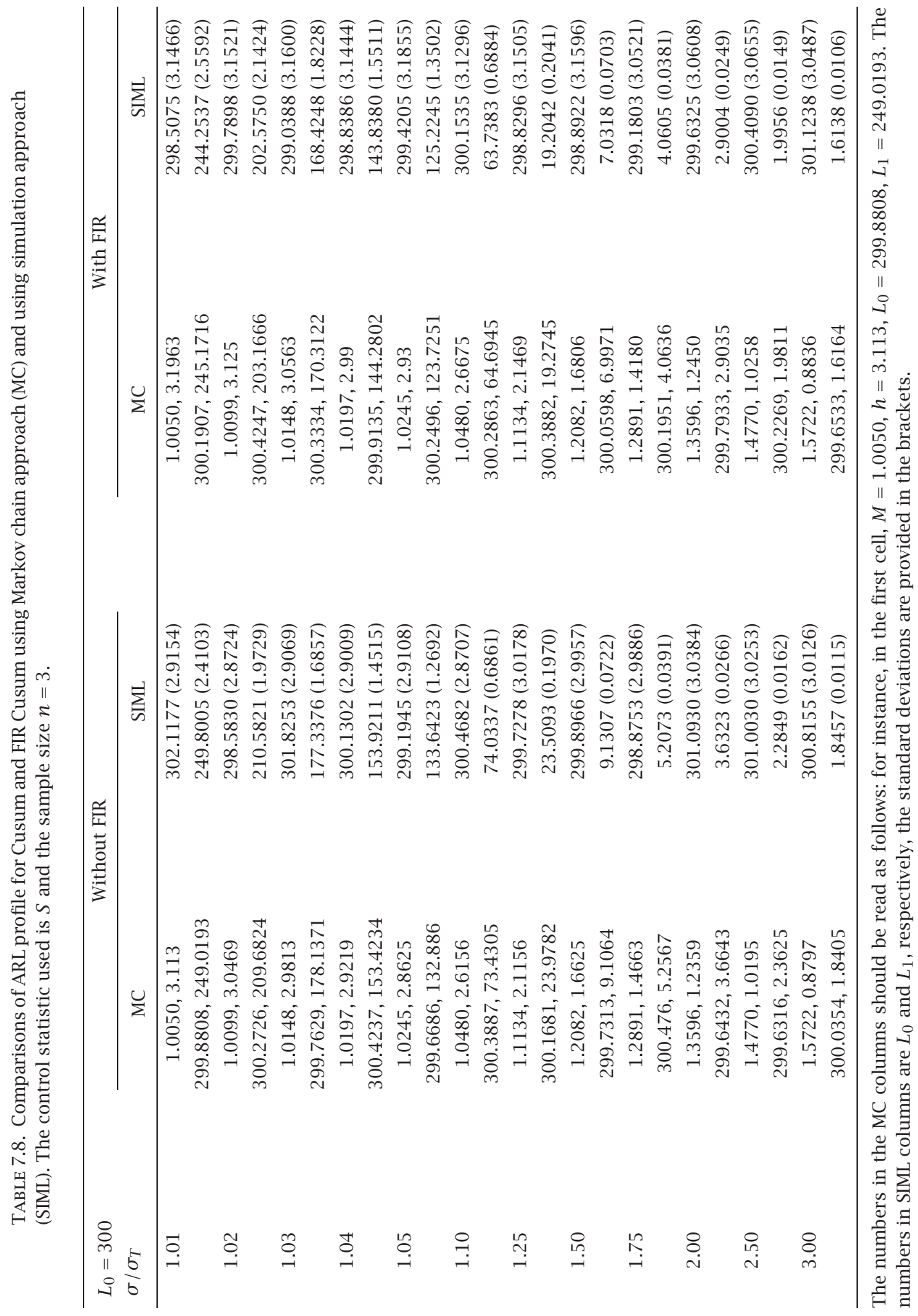




\section{Appendix}

We evaluate

$$
\int_{a}^{b} f(\lambda) d \lambda
$$

where $\lambda_{i}=V_{j}-M, a=(j-1) w-0.5 w$, and $b=(j-1) w+0.5 w$.

By definition $Y=n S^{2} / \sigma^{2}$ and $V=\left(k^{2} Y\right)^{\delta}$. Therefore, $\lambda=\left(k^{2} Y\right)^{\delta}-M$, where $Y=$ $n S^{2} / \sigma^{2} \sim X_{n-1}^{2}$, that is,

$$
f(y)=\frac{1}{\Gamma((n-1) / 2) 2^{(n-1) / 2}} y^{(n-1) / 2-1} \exp ^{-y / 2}, \quad 0 \leq y \leq \infty .
$$

Substituting $y=(\lambda+M)^{1 / \delta} / k^{2}$ into (A.2), and using the transformation formula for the probability density function of $y$, we have

$$
\int_{a}^{b} f(\lambda) d \lambda= \begin{cases}\int_{c}^{d} \frac{1}{\Gamma((n-1) / 2) 2^{(n-1) / 2}}\left\{\frac{(\lambda+M)^{1 / \delta}}{k^{2}}\right\}^{(n-1) / 2-1} & \\ \quad \times \exp \left\{\frac{-1}{2} \frac{(\lambda+M)^{1 / \delta}}{k}\right\} \frac{1}{k^{2} \delta}\left\{k^{2} \frac{(\lambda+M)^{1 / \delta}}{k^{2}}\right\}^{(1-\delta)} d \lambda, & \text { if }-M \leq \lambda \leq \infty, \\ 0, & \text { otherwise. }\end{cases}
$$

Defining

$$
u=\frac{(\lambda+M)^{1 / \delta}}{k^{2}} .
$$

Then (A.3) reduces to

$$
\int_{a}^{b} f(\lambda) d \lambda=\int_{c}^{d} \frac{1}{\Gamma((n-1) / 2) 2^{(n-1) / 2}} u^{(n-1) / 2-1} \exp \left(\frac{-u}{2}\right) d u=\int_{c}^{d} f(u) d u,
$$

where

$$
c=\frac{(a+M)^{1 / \delta}}{k^{2}}, \quad d=\frac{(b+M)^{1 / \delta}}{k^{2}}, \quad u \sim \chi_{(n-1)}^{2}
$$

\section{REFERENCES}

[1] M. A. Abdollahian, Optimal continuous inspection schemes, Ph.D. thesis, University College, Cardiff, UK, 1982.

[2] P. Bauer and P. Hackl, The use of MOSUMS for quality control, Technometrics 20 (1978), no. $4,431-436$.

[3] _ An extension of MOSUM technique for quality control, Technometrics 22 (1980), no. $1,1-7$.

[4] D. Brook and D. A. Evans, An approach to the probability distribution of cusum run length, Biometrika 59 (1972), no. 3, 539-549.

[5] T. C. Chang and F. F. Gan, A cumulative sum control chart for monitoring process variance, Journal of Quality Technology 27 (1995), no. 2, 109-119.

[6] S. V. Crowder and M. D. Hamilton, An EWMA for monitoring a process standard deviation, Journal of Quality Technology 24 (1992), no. 1, 12-21.

[7] R. Domangue and S. C. Patch, Some omnibus exponentially weighted moving average statistical process monitoring schemes, Technometrics 33 (1991), no. 3, 299-313.

[8] W. D. Ewan and K. W. Kemp, Sampling inspection of continuous processes with no autocorrelation between successive results, Biometrika 47 (1960), 363-380. 
[9] F. F. Gan, An optimal design of CUSUM quality control charts, Journal of Quality Technology 23 (1991), no. 4, 279-286.

[10] D. M. Hawkins, A CUSUM for a scale parameter, Journal of Quality Technology 13 (1981), no. 4, 228-231.

[11] J. M. Lucas and R. B. Crosier, Fast initial response for CUSUM quality control schemes: Give your CUSUM a head start, Technometrics 24 (1982), no. 3, 199-205.

[12] J. M. Lucas and M. S. Saccucci, Exponentially weighted moving average control schemes: properties and enhancements, Technometrics 32 (1990), no. 1, 1-29.

[13] J. F. MacGregor and T. J. Harris, The exponentially weighted moving variance, Journal of Quality Technology 25 (1993), no. 2, 106-118.

[14] G. V. Moustakides, Optimal stopping times for detecting changes in distributions, Ann. Statist. 14 (1986), no. 4, 1379-1387.

[15] C. H. Ng and K. E. Case, Development and evaluation of control charts using exponentially weighted moving average, Journal of Quality Technology 21 (1989), 242-250.

[16] E. S. Page, Continuous inspection schemes, Biometrika 41 (1954), 100-115.

[17]_ Controlling the standard deviation by CUSUM and warning lines, Technometrics 5 (1963), no. 3, 307-315.

[18] J. G. Ramirez, Sequential methods in statistical process monitoring, Ph.D. thesis, University of Wisconsin-Madison, Wisconsin, 1989.

[19] G. A. Regula, Optimal CUSUM procedure to detect a change in distribution for the gamma family, Ph.D. thesis, Case Western Reserve University, Ohio, 1976.

[20] M. R. Reynolds Jr., R. W. Amin, and J. C. Arnold, CUSUM charts with variable sampling intervals, Technometrics 32 (1990), no. 4, 371-396.

[21] R. J. Rowlands, A. B. J. Nix, M. A. Abdollahian, and K. W. Kemp, Snub-nosed V-mask control schemes, The Statistician 31 (1982), no. 2, 1-10.

[22] W. H. Woodall, The distribution of the run length of one-sided CUSUM procedures for continuous random variables, Technometrics 25 (1983), no. 3, 295-301.

[23] A. W. Wortham and L. J. Ringer, Control via exponential smoothing, The Logistics Review 7 (1971), 33-41.

S. Poetrodjojo: Department of Statistics And Operation Research, Royal Melbourne Institute of TeChNOlogy, GPO Box 2476 V, Melbourne Victoria 3001, Australia

M. A. Abdollahian: Department of Statistics AND Operation Research, Royal MelBOURne InSTITUTE of TECHNOLOGY, GPO BoX 2476 V, MElbourne ViCTORIA 3001, Australia

Narayan C. Debnath: Computer Science Department, Winona State University, WINONA, MN 55987, USA 


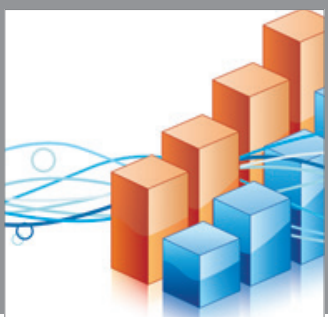

Advances in

Operations Research

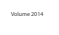

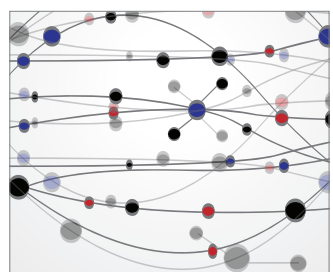

\section{The Scientific} World Journal
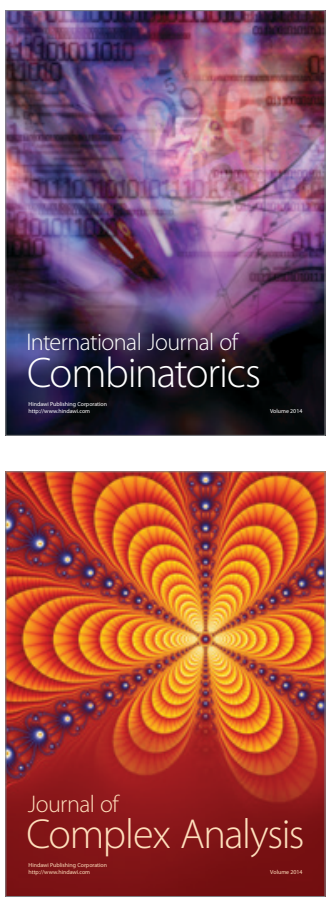

International Journal of

Mathematics and

Mathematical

Sciences
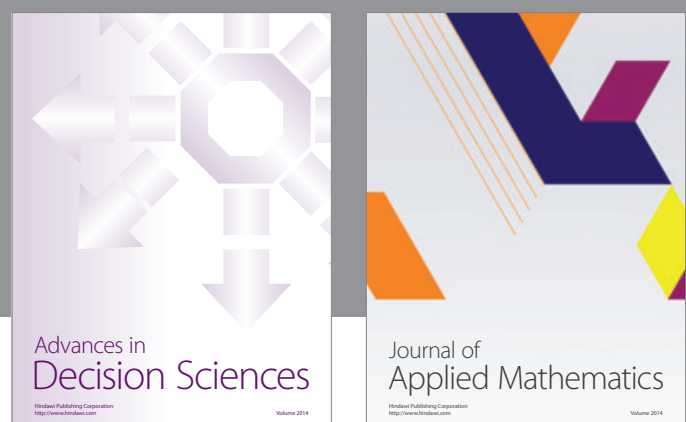

Journal of

Applied Mathematics
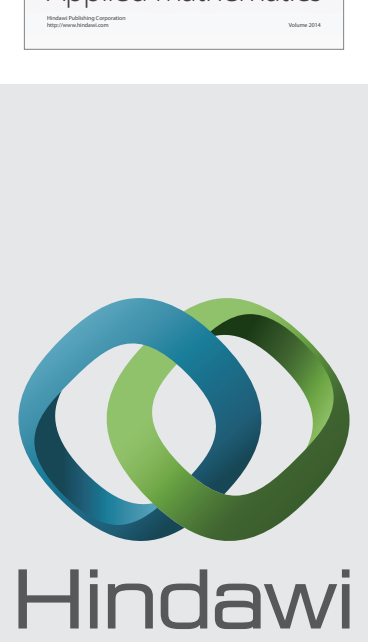

Submit your manuscripts at http://www.hindawi.com
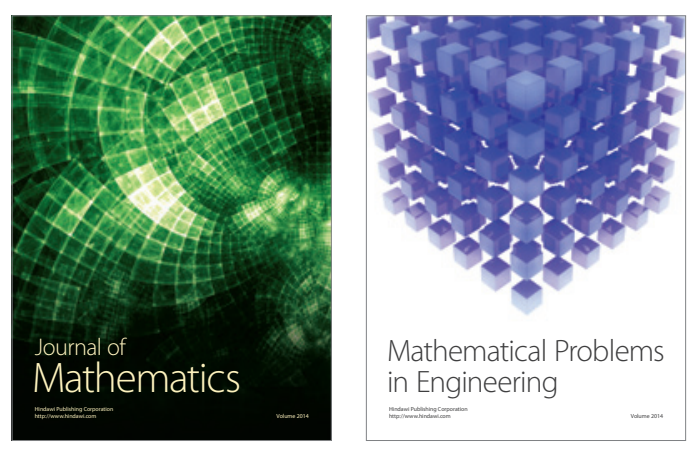

Mathematical Problems in Engineering
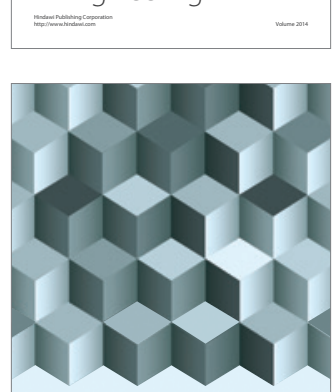

Journal of

Function Spaces
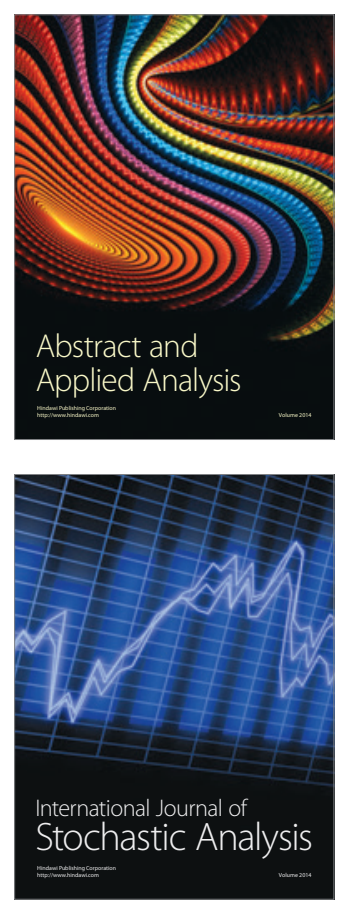

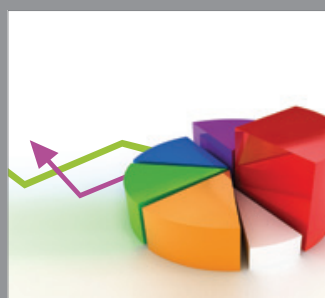

ournal of

Probability and Statistics

Promensencen
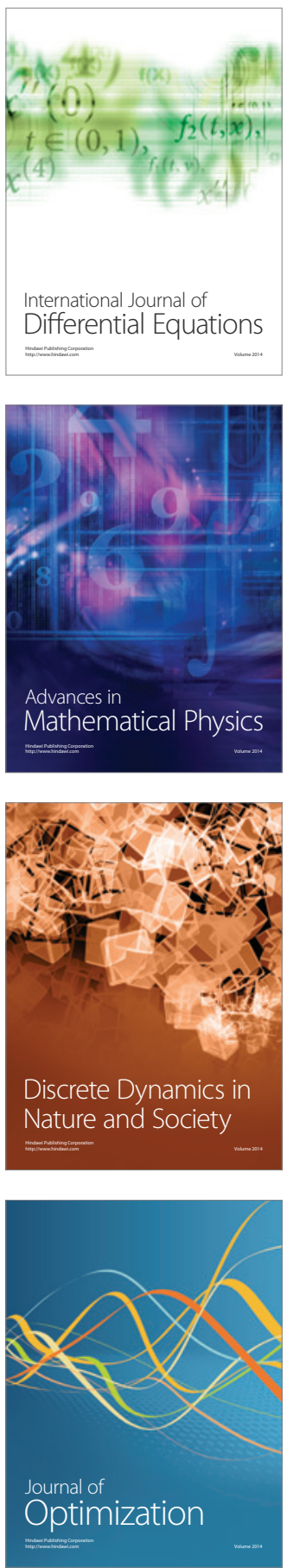